\title{
Electrostatics can be helpful in figuring out the spread through air of infectious diseases
}

\author{
Francesco Lattarulo \\ Dipartimento di Ingegneria Elettrica e dell'Informazione \\ Politecnico di Bari \\ Via Orabona, 4 - 70125 Bari (Italy) \\ E-mail address: francesco.lattarulo@poliba.it
}

\begin{abstract}
A physical model is here proposed as a speculative but robust attempt to understand a possible mechanism of contamination. An amount of micron-sized droplet nuclei, also carrying net charge with suitable polarity, are expected to be electro-dynamically involved in a mechanism originated by the static, thus natural and unperceivable, electrification of exposed subjects.
\end{abstract}

Keywords: Triboelectrification; human body model; airborne particulate; COVID-19.

\section{Introduction}

The in-progress pandemic pattern and incidence of COVID-19 are leading to interpret the ambient air conditions as a pivotal influencing factor [1], [2], [3]. The present investigation goes deeper into the subject through a line of reasoning that brings into play the static electricity normally affecting a human body (HB) [4]. The surrounding electric field, which thus comes to 
be generated, has been estimated as being large enough for an amount of suspended droplet nuclei carrying a net charge (see, for example, [5], [6]) to be driven towards the $\mathrm{HB}$, with a special focus on the frontal side of the head. Predictably, the triboelectric genesis, intended as the prerequisite for the capture of hazardous airborne particles by Coulombian forces, is strongly dependent on the ambient humidity, in turn often related to temperature, owing to the mechanism that will be carefully examined in Sect. 2. Among others, it is pointed out in Sect. 3 that the gender-based different vulnerability to COVID-19 [7], a feature which is still being monitored, finds support in the present framework. The concluding remarks provided in Sect. 4 are aimed at recommending appropriate safeguards, as those already put in place in totally different situations for a while already.

\section{The model}

\subsection{Charged human body as a source of driving electric field}

The human body electric potential (HBEP) is a consequence of the triboelectric effect mainly due to the shoe sole-floor contact. This is a typical precondition for the often referred to as electrostatic discharge (ESD) to occur. In the hypothetical disregard of suitable protective measures largely standardized for a while, the ESD is ultimately responsible of malfunction or definitive failure of electrically loop-connected and/or electromagnetically coupled sensitive electronics. The ESD severity is in direct proportion to the HBEP which amounts to $n_{v} \cdot 1 \mathrm{~V}$, with the number $n_{v}$ in the most usual range of $10^{3}$ to $10^{4}$. It is also important to bear in mind that 
the $\mathrm{HB}$ is subject to an unceasing and unintentional succession of ESD events and recharging phases, so that the HBEP can be deemed as being an inadvertent and natural condition that holds roughly stable in a daily timescale. According to a theoretical model [8], the HB is figuratively represented as an articulate multi-spheroidal assembly (the multi-sphere predecessor is described in [4]) composed by 11 prolate and oblate perfect conductors individually tailored to represent basic anatomical blocks and collectively interconnected in a manner that various postures can be manageably mimicked (and imaged, owing to the presence of the conducting plane). The value of the adopted method consists in providing a final circuit representation of the $\mathrm{HB}$ raised at an assigned potential, termed here as HBEP, from ground. The circuit is made of as many ground capacitances in parallel, thus connected to a common voltage source ensuring the application of a charge voltage $\mathrm{V}$ determined by, indeed identifying with, the HBEP itself. The lack of stray capacitances in that simple network finds answer within the special features of the theoretical method adopted, in the sense that each partial capacitance already takes into account mutual electrostatic couplings, even those with nearby walls, furniture and other people. It follows that any given anatomical block can be taken as being in isolation and driven by the voltage V. Of course, the above set of partial capacitances are subject to change, each in its own way, in function of posture and external influences. If the attention is focused on the HB head and, hence, on the capacitive counterpart, it has been proved that it can only fall within the restricted order of some picofarads under all realistic circumstances $(7 \mathrm{pF}$ could be a representative value for an adult subject). An estimate of the induced nearby electric field $\mathrm{E}$ can be calculated 
by using the simple formula $\mathrm{E}=\mathrm{V} /(\mathrm{r}+\mathrm{d})$, with $\mathrm{r}=0.106 \mathrm{~m}$ and $\mathrm{d} \geq 0$ respectively standing for the radius of the sphere equivalent in volume to the HB head and the distance of the outer field point from the spherical surface. In fact, it seems permissible at this stage to replace the slightly prolate spheroid, as an original substitute of the head, with the equivalent sphere. The substantive result is $E=n_{E} \cdot 1 \mathrm{~V} / \mathrm{m}$, bearing in mind that $\mathrm{E}$ is a scalar function of position and $\mathrm{V}=\mathrm{n}_{\mathrm{v}} \cdot 1 \mathrm{~V}$. Therefore, the multiplicative factor $n_{E}$ is in the characteristic range of $10^{4}$ to $10^{5}$ that reduces to one-tenth for increasing $\mathrm{d}$ from 0 to $1 \mathrm{~m}$.

\subsection{Transport mechanism in still air}

Once respiratory droplets are expelled by speaking, coughing and sneezing, and then are disseminated in the ambient airspace, they could be ultimately involved in an electrostatically driven transport mechanism instead of settling eventually to the floor. The flow in question consists of drifting micro-droplets, having suitable net charge, under the thrust of Coulombian forces resisting the obstruction of buffer air molecules. A special class of respiration-originated airborne is in fact represented by droplet nuclei which are argued to drift in considerable quantity, determined by their polarity and restricted aerodynamic diameter $\left(\mathrm{d}_{\mathrm{c}} \leq 10 \mu \mathrm{m}\right.$; most commonly in the range of $1 \mu \mathrm{m}$ ), towards the human face acting as a source for the externally driving electric field; see details later. The described scenario is harmful precisely because the droplet nuclei contain infectious agents and might at most settle to ground slowly under the force of gravity [5]. In fact, their diameter has been reduced, in response to evaporation of the aqueous content [9], to the extent that the residuals are having to float longer. The 
latter performance is the result of collisions with the random thermal motion of air molecules. On the other hand, a plentiful number of biological particles in the airborne state can bear a net charge $\mathrm{q}_{\mathrm{c}}=\mathrm{e}^{\cdot} \mathrm{n}_{\mathrm{c}}$, where $\mathrm{e}=1 \cdot 10^{-}$ ${ }^{19} \mathrm{C}$ is the elementary charge and the number $\mathrm{n}_{\mathrm{c}}$ is in the range of 0 to $10^{3}$. If the droplet core is occupied by a pathogenic micro-organism, which often carries its own natural charge, then it is expected that an important extracharge is deposited onto its surface as a result of desiccation. This it is exactly the case for droplet nuclei, so that setting $\mathrm{n}_{\mathrm{c}}$ of some unities multiplied by $10^{3}$ becomes a realistic condition for an amount of droplet nuclei carrying net charge. For the sake of comparison, consider that nonbiological aerosols acquire charge in ambient air by a composite diffusional and field charging mechanism due to collisions with, and attachment to, small ions having a diameter of about $1 \mathrm{~nm}$ [10]. The number of charges an aerosol of such a species can collect hardly goes beyond the unit [11]. This happens because the actual Coulomb barrier largely exceeds the thermal ion energy.

All things considered, the droplet nuclei individually undergo a driving force whose modulus $\mathrm{F}_{\mathrm{E}}=\mathrm{q}_{\mathrm{c}} \cdot \mathrm{E}$ is in the range $10^{-13} \div 10^{-12} \mathrm{~N}$ at a distance $\mathrm{d}$ $=1 \mathrm{~m}$ from the HB head, and grows tenfold in the critical case of close proximity to the $\mathrm{HB}$ face $(\mathrm{d} \approx 0)$. If that biological airborne is approximately identified with a unit density sphere (mass density $\delta=1 \mathrm{~g} / \mathrm{cm}^{3}$ ), then some simple algebra allows verification that the impressed electrostatic force is one to two orders stronger than the force of gravity $\mathrm{F}_{\mathrm{G}}=\pi \cdot \mathrm{d}_{\mathrm{c}}^{3} \cdot \delta \cdot \mathrm{g} / 6$, with $\mathrm{g}$ being the gravitational deceleration. This result is in line with the previously discussed negligible transversal disturbance from the gravitational 
component. The key importance of this comparison urges further examination such as that which directly involves drift velocities in substitution of the causative pushing forces $F_{E}$ and $F_{G}$. On the basis of this position, let $\mathrm{v}_{\mathrm{E}}$ and $\mathrm{v}_{\mathrm{G}}$ be the respective velocities of the droplet nuclei simultaneously subject to electrostatic attraction towards the HB head and gravitation. Governing equations for particulates in airborne state are

$$
\begin{gathered}
\mathrm{v}_{\mathrm{E}}=\frac{\mathrm{q}_{\mathrm{c}}}{3 \cdot \pi \cdot \mathrm{d}_{\mathrm{c}} \cdot \eta} \cdot \mathrm{E} \\
\mathrm{v}_{\mathrm{G}}=\frac{\mathrm{g} \cdot \delta \cdot \mathrm{d}_{\mathrm{c}}}{18 \cdot \eta}
\end{gathered}
$$

The quantity embodied in Eq. (1) as a fraction multiplied by E represents the mobility of the drifting droplet nuclei - these rather meant like forming a specific charge-bearing species -, whereas Eq. (2) applies to its settling in relation to the in-tandem ponderable aspect of the droplet identity. Both formulas tacitly assume that the aerial fluid is a monomolecular gas in thermal equilibrium with negligible Knudsen number [12]. This implies that the buffer gas behaves macroscopically as a continuum because $\mathrm{d}_{\mathrm{c}}$ largely exceeds the mean free path of air molecules. The shared dynamic viscosity $\eta$ is also evocative of the macroscale behaviour mentioned above. Eqs. (1) and (2) needs to be generalised by the introduction of a multiplicative factor larger than 1 , notably the Cunningham slip correction factor, when the aforementioned condition for the Knudsen number does not hold; see, for example, [13]. A simple computational exercise make it possible to become aware that the mobility of the droplet under consideration can amount to $10^{-}$ $7 \div 10^{-6} \mathrm{~m}^{2} /(\mathrm{V} \cdot \mathrm{s})$, especially by virtue of the remarkably large value of $\mathrm{q}_{\mathrm{c}}$ which is permissible, and distinctive, for biological airborne. Under the 
influence of the E-field, $v_{E}$ is therefore prone to exceed $v_{G}$ as much as two orders of magnitude, the latter velocity being close to $10^{-4} \mathrm{~m} / \mathrm{s}$. It is worth parenthetically stressing that rearranging Eqs. (1) and (2) gives a ratio, $\mathrm{v}_{\mathrm{E}} / \mathrm{v}_{\mathrm{G}}$, indistinguishable from $\mathrm{F}_{\mathrm{E}} / \mathrm{F}_{\mathrm{G}}$. This result is not so surprising because the driving forces $F_{E}$ and $F_{G}$ are counteracted by drag forces due to the common buffer gas.

\section{Discussion}

The above description provides unique insight into the role that the natural $\mathrm{HB}$ electrification performs in raising the risk of infection with a serious chance of death. On the concern, some pertaining considerations are summarized below.

It is believed that the HB can electrostatically capture a not inconsiderable, even though hardly predictable, amount of droplet nuclei previously disseminated in calm air. The value of sub-Sect. 2.2 consists in providing convincing arguments to figure out how just the head can collect within a pair minutes the majority of airborne pathogens carrying a polarity opposed to the HBEP and contained, say, in a surrounding spherical airspace of $1 \mathrm{~m}$ radius. Static electricity is a nearly perpetual physical accident for the $\mathrm{HB}$, for which reason it cannot escape, in the lack of effectively screening and ready-to-wear accessories (if existing special mitigation measures are unavailable), from being polluted and then easily contaminated in shared rooms. Things get worse when two or more individuals are in close proximity and the electrostatic scenario that accidentally unfolds is such that they are mutually subject to enhanced collecting effects. In the simpler case of two individuals, say, $\mathrm{HB}^{+}$and $\mathrm{HB}^{-}$, it cannot be ruled out that they happen 
to bear charge with positive and negative polarity, respectively. The obvious consequence is that negatively (positively) charged surrounding droplets are pushed faster and in greater number towards the face of $\mathrm{HB}^{+}\left(\mathrm{HB}^{-}\right)$under the action of the enhanced background electric field.

According to the model discussed in in sub-Sect. 2.1, similar consideration could be applied to the remaining blocks in which the HB has been partitioned. This is an additional, useful observation since environmental charge-bearing droplet nuclei may be also deposited on the HB's clothing and exposed parts, if there were any, even as a result of rubbing seats and beddings.

The collecting mechanism under examination also provides natural insight into why suspected correlations between infection spreading and ambient air conditions do make sense [1]. In particular, it has been argued that the spreading attenuates as humidity increases, thus in considerable accordance with the static-electricity-based model claimed here. It is well established that the HBEP remarkably decreases, according to linear trends as those displayed in [14], as the relative humidity enhances. This behaviour is explained because the usually low surface conductivity of the insulating materials acting as triboelectric source tend to increase when they are exposed to moist environment. Importantly, this is the typical case of anyone who wears shoes while walking. The expected result is that leakage over surface routes, no longer insulating sufficiently, of charge released by triboelectric effect, increases disproportionately. This can only happen at the expense of the charge amount that succeeds in distributing on the surface of the badly insulated HB, with consequent scaling-down of the related HBEP 
rising. In contrast, humidity seems not to produce direct consequences on the moist ambient air. In particular, even massive moisture turns out to have an insignificant effect on the ambient permittivity, so that the HB capacitance remains almost unchanged. From the present perspective, the moisture-dependence of charge leakage that makes HBEP decline could therefore result in a pleasingly reduced action of the HB's face as a critical electrostatic collector of pathogens.

An additional factor that must not be overlooked is that the non-zero surface resistivity of the imperfectly conducting $\mathrm{HB}$ is a further feature that concurs, as a dual partner of the non-infinite resistivity of the imperfectly insulating shoes, in reducing the HBEP and related effects. In fact, available data lead to argue some sort of relationship between the ESD peak with the HB's skin resistance and/or chemistry rather than geometry (shape and dimensions) [15]. The skin resistance also slows down the charging law that governs the transitory distribution of charge over the HB surface, thus prior to a possibly definitive deposition in electrostatic equilibrium. Indeed, this steady state could not be achieved when an unpredictable and abrupt ESD does prevent full charging, hence, the HBEP to become as large as it can theoretically be. In physiological terms, it follows that such a potential is expected to significantly increase as the surface conductivity of the HB increases. As a reflection of this matter, due account is here taken of gender differences, with the exception of the dimorphism for the same reasons that have already stressed before. In short, more men than women seem to be the most suitable candidates to exhibit upper HBEP, very likely because the former human class shall be faster than the latter in the HB charging process due to its 
higher surface conductivity. For example, the observed sweating response to physical activity - with $30 \%$ to $40 \%$ more for men -, the thicker skin with the consequential result of a proportionally larger amount of water contained therein -, combined with more vascularized upper dermis [16], are really favourable preconditions for the HBEP to rise faster towards higher levels. It is possible that even some sex-specific physiological differences in body composition and evolutionary reasons [17], could contribute to improve mostly for men the efficiency of their static electrification. The tangible consequence of the above is that a proportionally larger amount of electrostatically driven droplet nuclei may be inhaled, or penetrate through the mouth and eyes of male subjects, with consequent enhanced fatal outcomes for the male population.

\section{Concluding remarks}

Given the lack of a vaccine, at least for the time being, the implementation of preventative measures founded on an ever broader interdisciplinary knowledge on infection spreading cannot but be a recommended practice. The present investigation intends to be incremental by soliciting appropriate attention to this issue. In this regard, it should be noted that mitigating provisions for charge buildup in critical or emergency situations to be prevented, or effectively dissipated, are nowadays largely referred to in the realm of the EMC (electromagnetic compatibility).

\section{References}

[1] M. M. Sajadi, P. Habibzadeh, A. Vintzileos, S. Shokouhi, F. Miralles-Wilhelm, A. Amoroso, Temperature, Humidity and Latitude Analysis to Predict Potential Spread and Seasonality for COVID-19 (March 5, 2020). https://ssrn.com/abstract=3550308; http://dx.doi.org/10.2139/ssrn.3550308 
[2] Q. Bukhari, Y. Jameel, Will Coronavirus Pandemic Diminish by Summer? March 17, 2020. https://ssrn.com/abstract=3556998; http://dx.doi.org/10.2139/ssrn.3556998

[3] J. Wang, K. Tang, K. Feng, Kai and W. Lv, High Temperature and High Humidity Reduce the Transmission of COVID-19 (March 9, 2020). https://ssrn.com/abstract=3551767; http://dx.doi.org/10.2139/ssrn.3551767

[4] V. Amoruso, M. Helali, F. Lattarulo, An improved model of man for ESD applications", J. Electrostatics, 49 (2000) 225-244.

[5] R. Tellier, Y. Li, B. Y. Cowling, J. W. Tang, Recognition of aerosol transmission of infectious agents: a commentary, 19:101 (2019) 1-9 https://doi.org/10.1186/s12879019-3707-y

[6] G. Mainelis, K. Willeke, P. Baron, T. Reponen, S. Y. Grinshpun, R. L. Gorny, S. Trakumas, Electrical charges on airborne microorganisms, Aerosol Sci. 32 (2001) 1087-1110.

[7] Global Health 50/50, Sex, gender and COVID-19, http://globalhealth5050.org/covid19/\#

[8] V. Amoruso, F. Lattarulo, Diakoptic approach to EMC problems involving the human body, in: Electromagnetic Compatibility in Power Systems, F. Lattarulo Editor, Elsevier, Oxford (UK), 2007, 133-164.

[9] X. Xie, Y. Li, H. Sun, L. Liu, Exhaled droplets due to talking and coughling, J. R. Soc. Interface, 6 (2009) S703-S714 doi:10.1098/rsif.2009.0388.focus.

[10] T. D. Bracken, Small air ion properties, in Air Ions: Physical and Biological Aspects, J. M. Charry, R. Kavet Eds., Ch. 1, CRC-Press, 1987.

[11] B. M. Smirnov, Cluster Processes in Gases and Plasmas, Wiley-VCH, Weinheim (2010) 331-354.

[12] F. Lattarulo, V. Amoruso, Filamentary Ion Flow - Theory and Experiments, IEEE Press-Wiley, Hoboken (NJ), 2014.

[13] R. W. Welker, Basics and sampling of particles for size analysis and identification, in: Developments in Surface Contamination and Cleaning, R. Kohli and K. L. Mittal Eds., Elsevier, Amsterdam, 2012, 1-80.

[14] P. Wilson, The Circuit Designer's Companion, Elsevier, Amsterdam, 2012, 334340, 374-376. 
[15] M. A. Kelly, G. E. Servais, T. V. Pfaffenbach, An investigation of human body electrostatic discharge, ISTFA'93, Los Angeles, 15-19 Nov. 1993, 167-173.

[16] P. U. Giacomoni, T. Mammone, M. Teri, Gender-linked differences in human skin, J. Dermatological Sci., 55 (2009) 144-149.

[17] S. Kirchengast, Gender differences in body composition from childhood to old age: an evolutionary point of view. J. Life Sci. 2(1) (2010). 